\title{
Achieving robustness through coarse space enrichment in the two level Schwarz framework.
}

\author{
Nicole Spillane ${ }^{1,2}$, Victorita Dolean ${ }^{3}$, Patrice Hauret ${ }^{2}$, Frédéric Nataf ${ }^{1}$, Clemens \\ Pechstein $^{4}$, and Robert Scheichl ${ }^{5}$
}

Key words: coarse space, heterogeneous coefficients, robustness

As many domain decomposition methods the two level Additive Schwarz method may suffer from a lack of robustness with respect to coefficient variation in the underlying set of PDEs. This is the case in particular if the partition into subdomains is not aligned with all jumps in the coefficients. Thanks to the theoretical analysis of two level Schwarz methods (see [11] and references therein) this lack of robustness can be traced back to the so called stable splitting property (already in [4]). Following the same ideas as in the pioneering work [1] we propose to solve a generalized eigenvalue problem in each subdomain which identifies which vectors are responsible for slow convergence. The spectral problem is specifically chosen to separate components that violate the stable splitting property. These vectors are then used to span the coarse space which is taken care of by a direct solve while all remaining components can be resolved on the subdomains. The result is a preconditioned system with a condition number estimate that does not depend on the number of subdomains or any jumps in the coefficients. We refer to this method as GenEO for Generalized Eigenproblems in the Overlaps. It is closely related to the work of [2] where the same strategy leads to a different eigenproblem and different condition number estimate (which also does not depend on the jumps in the coefficients or on the number of subdomains). A full theoretical analysis of the two level Additive Schwarz method with the GenEO coarse space (first briefly introduced in [8]) is given in [7]. Here our purpose is to show the steps leading from the abstract Schwarz theory to the choice of our generalized eigenvalue problem (5). In the first section we introduce the rather wide range of problems to which the method applies and give the classical two-level Schwarz condition number estimate in the abstract framework (again, see [11] and references therein). In the second section we work to make this condition local (on each subdomain), identify the GenEO generalized eigenproblem and state our main result (Theorem 2). Finally in the third section we illustrate the result numerically.

Laboratoire Jacques-Louis Lions, CNRS UMR 7598, Université Pierre et Marie Curie, 75005 Paris, France - Manufacture des Pneumatiques Michelin, 63040 Clermont-Ferrand, Cedex 09, France. - Laboratoire J.-A. Dieudonné, CNRS UMR 6621, Université de Nice-Sophia Antipolis, 06108 Nice Cedex 02, France. · Institute of Computational Mathematics, Johannes Kepler University, Altenberger Str. 69, 4040 Linz, Austria. · Department of Mathematical Sciences, University of Bath, Bath BA27AY, UK. 


\section{Problem Setting}

Given a finite dimensional Hilbert space $V_{h}$, a continuous and coercive bilinear form $a: V_{h} \times V_{h} \rightarrow \mathbb{R}$ and a right hand side $f \in V_{h}^{\prime}$ we consider the following problem. Find $v \in V_{h}$ such that $a(v, w)=\langle f, w\rangle$ for all $w \in V_{h}$. Then given a basis for $V_{h}$ we can derive a linear system $\mathbf{A v}=\mathbf{f}$.

Assumption: The following assumption is needed on the bilinear form: $a$ is given through positive semi definite element matrices $\left\{a_{\tau}\right\}_{\tau \in \mathscr{T}_{h}}$ where $\mathscr{T}_{h}$ is a mesh on the computational domain $\Omega$ underlying $V_{h}$. Our method can also be defined for abstract elements and degrees of freedom as in [7] but here we focus on PDEs and prefer this more intuitive point of view.

The reason why we require this assumption is so that we may define, for any subset $D$ which is resolved by the mesh, the following local bilinear form:

$$
a_{D}(v, w):=\sum_{\tau \subset D} a_{\tau}\left(v_{\mid \tau}, w_{\mid \tau}\right)
$$

The Additive Schwarz method is based on an overlapping partition $\left\{\Omega_{j}\right\}_{j=1}^{N}$ of $\Omega$ where each $\Omega_{j}$ is resolved by the mesh. On each of these subdomains, we denote the space of functions supported in $\Omega_{j}$ by: $V_{h, 0}\left(\Omega_{j}\right):=\left\{v_{\mid \Omega_{j}}: v \in V_{h}, \operatorname{supp}(v) \subset \Omega_{j}\right\}$.

An important role is played by the extension operator $R_{j}^{\top}: V_{h, 0}\left(\Omega_{j}\right) \rightarrow V_{h}$ which returns the extension by zero of a function $v \in V_{h, 0}\left(\Omega_{j}\right)$ to $\Omega$. The adjoint of $R_{j}^{\top}$ is the restriction operator $R_{j}: V_{h}^{\prime} \rightarrow V_{h, 0}\left(\Omega_{j}\right)^{\prime}$ defined by $\left\langle R_{j} g, v\right\rangle=\left\langle g, R_{j}^{\top} v\right\rangle$, for $v \in V_{h, 0}\left(\Omega_{j}\right), g \in V_{h}^{\prime}$. Let $\mathbf{R}_{j}$ be the matrix representation of $R_{j}$. This is a boolean matrix. Then the one level Additive Schwarz preconditioner is defined simply based on these interpolation operators as $\mathbf{M}_{A S, 1}^{-1}:=\sum_{j=1}^{N} \mathbf{R}_{j}^{\top} \mathbf{A}_{j}^{-1} \mathbf{R}_{j}$ where $\mathbf{A}_{j}:=\mathbf{R}_{j} \mathbf{A} \mathbf{R}_{j}^{\top}$ are the local problem matrices.

In other words, the one level Schwarz preconditioner approximates the inverse of the global matrix $\mathbf{A}^{-1}$ by a sum of local inverses $\mathbf{A}_{j}^{-1}$. The method is known to converge [11] as long as the subdomains and finite element spaces are chosen so that $V_{h}=\sum_{j=1}^{N}\left[R_{j}^{\top} V_{h, 0}\left(\Omega_{j}\right)\right]$. In some sense this ensures that the local subdomains are overlapping enough. The drawback of the one level Schwarz method is that its convergence rate depends on the number of subdomains and thus scales poorly for large problems. The introduction of a coarse space is a, by now classical, way of weakening this dependence. Having chosen the coarse space $V_{H}$ and an interpolation operator $R_{H}^{\top}: V_{H} \rightarrow V_{h}$, the two-level Additive Schwarz preconditioner is the most simple two level method: it reads

$\mathbf{M}_{A S, 2}^{-1}:=\mathbf{R}_{H}^{\top} \mathbf{A}_{H}^{-1} \mathbf{R}_{H}+\sum_{j=1}^{N} \mathbf{R}_{j}^{\top} \mathbf{A}_{j}^{-1} \mathbf{R}_{j}, \quad \mathbf{A}_{H}:=\mathbf{R}_{H} \mathbf{A} \mathbf{R}_{H}^{\top}$ (Coarse problem matrix),

where $\mathbf{R}_{H}$ is the matrix representations of $R_{H}$. 
The following theorem is simply a reformulation of the results in Chapter 2 of the book by Toselli and Widlund [11] where the abstract Schwarz theory is presented. We refer to there for the proof.

Theorem 1 (Condition number in the abstract Schwarz theory). Let $k_{0}$ be the maximal degree of multiplicity of a point in $\Omega$ with respect to the partition into subdomains: $k_{0}=\max _{\mathbf{x} \in \Omega}\left(\#\left\{\Omega_{j}: 1 \leq j \leq N, \mathbf{x} \in \bar{\Omega}_{j}\right\}\right)$.

Assume that for a fixed constant $C_{0}$ there exists a stable splitting $\left(z_{H}, z_{1}, \ldots, z_{N}\right) \in$ $V_{H} \times V_{h, 0}\left(\Omega_{1}\right) \times \ldots \times V_{h, 0}\left(\Omega_{N}\right)$ of any $v \in V_{h}$ :

$$
v=R_{H}^{\top} z_{H}+\sum_{j=1}^{N} R_{j}^{\top} z_{j} ; \quad a\left(R_{H}^{\top} z_{H}, R_{H}^{\top} z_{H}\right)+\sum_{j=1}^{N} a\left(R_{j}^{\top} z_{j}, R_{j}^{\top} z_{j}\right) \leq C_{0}^{2} a(v, v) .
$$

Then the condition number of A preconditioned by the two level Additive Schwarz operator satisfies $\kappa\left(\mathbf{M}_{A S, 2}^{-1} \mathbf{A}\right) \leq\left(k_{0}+1\right) C_{0}^{2}$.

This theorem is the cornerstone of our method and we make our objective more precise thanks to these two remarks:

- The constant $k_{0}$ in the inequality does not depend on the number of subdomains but only on the geometry of the partition. For instance in two dimensions if a regular partition into rectangular subdomains is used then $k_{0}=4$ no matter what the total number of subdomains is. This means that the presence of $k_{0}$ in the estimate does not violate scalability.

- To make the theorem more precise, $C_{0}^{-2}$ is a lower bound for the eigenvalues of the preconditioned operator and $k_{0}+1$ is an upper bound. The upper bound holds and is sharp regardless of the choice of the (non empty) coarse space. For this reason we do not work to improve the upper bound and instead we will work only on the lower bound through the stable splitting assumption.

Now the question of making the method robust with respect to the number of subdomains and the coefficients in the PDEs reduces to the following problem:

Find a coarse space $V_{H}$ for which there exists a constant $C_{0}$ independent of the number of subdomains and the coefficients in the underlying set of PDEs such that any $v \in V_{h}$ admits a stable splitting (3) onto this coarse space and the local subspaces.

\section{From the abstract Schwarz theory to the GenEO coarse space}

The practical inconvenience of the stable splitting property is that it is not local. Reducing it to $N$ local problems relies on the following observation: there are two simple ways to get a local version of $v$, either with the restriction operator $R_{j} v$ which returns a function in $V_{h, 0}\left(\Omega_{j}\right)$ that is supported in $\overline{\Omega_{j}}$ or by restricting the domain 
of $v$ to $\Omega_{j}$ which we denote $v_{\mid \Omega_{j}}$. There is no immediate inequality between the global term $a(v, v)$ and any of the local terms $a_{\Omega_{j}}\left(R_{j} v, R_{j} v\right)$. However the alternative inequality $a(v, v) \geq a_{\Omega_{j}}\left(v_{\mid \Omega_{j}}, v_{\mid \Omega_{j}}\right)$ holds (and motivates the following lemma), since according to (1),

$$
a(v, v)=a_{\Omega}(v, v)=a_{\Omega_{j}}\left(v_{\mid \Omega_{j}}, v_{\mid \Omega_{j}}\right)+\underbrace{a_{\Omega \backslash \Omega_{j}}\left(v_{\mid \Omega \backslash \Omega_{j}}, v_{\mid \Omega \backslash \Omega_{j}}\right.}_{\geq 0}) .
$$

Lemma 1. Given $v \in V_{h}$, if there exists a splitting $v=z_{H}+z_{1}+\ldots+z_{N}$ such that each local component $(j=1, \ldots, N)$ satisfies $a\left(R_{j}^{\top} z_{j}, R_{j}^{\top} z_{j}\right) \leq C_{1} a_{\Omega_{j}}\left(v_{\mid \Omega_{j}}, v_{\mid \Omega_{j}}\right)$, then the splitting is stable in the sense of (3) for $C_{0}^{2}=2+C_{1} k_{0}\left(2 k_{0}+1\right)$.

Proof. Using the definition of $k_{0}$ we can bound the sum of the local contributions:

$$
\sum_{j=1}^{N} a\left(R_{j}^{\top} z_{j}, R_{j}^{\top} z_{j}\right) \leq C_{1} \sum_{j=1}^{N} a_{\Omega_{j}}\left(v_{\mid \Omega_{j}}, v_{\mid \Omega_{j}}\right) \leq C_{1} k_{0} a(v, v) .
$$

The bound for the energy of the coarse contribution follows from $R_{H}^{\top} z_{H}=v-$ $\sum_{j=1}^{N} R_{j}^{\top} z_{j}$ which implies $a\left(R_{H}^{\top} z_{H}, R_{H}^{\top} z_{H}\right) \leq 2 a(v, v)+2 a\left(\sum_{j=1}^{N} R_{j}^{\top} z_{j}, \sum_{j=1}^{N} R_{j}^{\top} z_{j}\right)$ and, by the definition of $k_{0}$ and the previous inequality,

$$
a\left(\sum_{j=1}^{N} R_{j}^{\top} z_{j}, \sum_{j=1}^{N} R_{j}^{\top} z_{j}\right) \leq k_{0} \sum_{j=1}^{N} a\left(R_{j}^{\top} z_{j}, R_{j}^{\top} z_{j}\right) \leq C_{1} k_{0}^{2} a(v, v) .
$$

Putting all of these estimates together ends the proof of the lemma.

Lemma 1 also explains why we think of the coarse space as the space of bad components. Indeed, it states that it is enough to check that an estimate holds on each of the local components $z_{j}$ of the splitting. Then this implies an estimate for the coarse component $z_{H}$ and in turn the stable splitting assumption is satisfied.

An important tool in building the GenEO coarse space is a family of partition of unity operators. The particularity of these partition of unity operators is that they are defined at the degree of freedom level. The main consequence is that when the partition of unity is applied to a function we do not need to reinterpolate into the finite element space as is classically the case in partition of unity spaces where an application of the partition of unity is a multiplication by a continuous function.

Definition 1 (Partition of unity). For each subdomain let $\operatorname{dof}\left(\Omega_{j}\right)$ be the set of degrees of freedom for which the associated basis function $\phi_{k}$ is supported in $\overline{\Omega_{j}}$ : $\operatorname{dof}\left(\Omega_{j}\right)=\left\{k ; \operatorname{supp}\left(\phi_{k}\right) \subset \overline{\Omega_{j}}\right\}$. Then for each degree of freedom $k=1, \ldots, n$ let $\left\{\mu_{j, k}\right\}_{\left\{j: k \in \operatorname{dof}\left(\Omega_{j}\right)\right\}}$ be a family of weights $\left(\mu_{j, k} \geq 1 \quad\right.$ and $\left.\quad \sum_{\left\{j: k \in \operatorname{dof}\left(\Omega_{j}\right)\right\}} \frac{1}{\mu_{j, k}}=1\right)$. Finally the local partition of unity operator for $v \in V_{h}$ written as $v=\sum_{k=1}^{n} v_{k} \phi_{k}$ is defined by

$$
\Xi_{j}\left(v_{\mid \Omega_{j}}\right):=\sum_{k \in \operatorname{dof}\left(\Omega_{j}\right)} \frac{1}{\mu_{j, k}} v_{k} \phi_{k \mid \Omega_{j}} .
$$


This definition gives rise to a few remarks:

- A possible choice for the weights in the definition of the partition of unity is to use the multiplicity of each degree of freedom (this is what we use in the numerical section): for any degree of freedom $k, 1 \leq k \leq n$, let $\mu_{k}$ denote the number of subdomains for which $k$ is an internal degree of freedom, i.e.

$$
\mu_{k}:=\#\left\{j: 1 \leq j \leq N \text { and } k \in \operatorname{dof}\left(\Omega_{j}\right)\right\} .
$$

Then let $\mu_{j, k}=\mu_{k}$ for every subdomain $j$ for which $k \in \operatorname{dof}\left(\Omega_{j}\right)$.

- Other more coefficient adapted choices similar to those in [3] could be made.

- The family of operators $\left\{\Xi_{j}\right\}_{j=1, \ldots, N}$ indeed forms a partition of unity since $\sum_{j=1}^{N} R_{j}^{\top} \Xi_{j}\left(v_{\mid \Omega_{j}}\right)=v$ for any $v \in V_{h}$. This provides an obvious splitting of $v$ onto the local subspaces.

- The partition of unity operator $\Xi_{j}$ takes the restriction of a function to subdomain $\Omega_{j}$ and returns a function in $V_{h, 0}\left(\Omega_{j}\right)$ (which is supported in $\bar{\Omega}_{j}$ ).

- If a degree of freedom $k$ belongs to only one subdomain $j$ then $\mu_{j, k}=1$ and $\left(\Xi_{j}\left(v_{\mid \Omega_{j}}\right)\right)_{k}=\left(v_{\mid \Omega_{j}}\right)_{k}$. This is the reason why the overlap plays a special role in the generalized eigenvalue problem which separates good and bad components. More detail is given in the proof of the final theorem.

Next we introduce the GenEO coarse space.

\section{Definition 2 (GenEO coarse space).}

1. For each subdomain $\Omega_{j}(1 \leq j \leq N)$, let the overlap be given by

$$
\Omega_{j}^{\circ}=\bigcup\left\{\tau \subset \overline{\Omega_{j}}: \exists j^{\prime} \neq j \quad \text { such that } \tau \subset \overline{\Omega_{j^{\prime}}}\right\} .
$$

2. For each $j=1, \ldots, N$, solve the following generalized eigenvalue problem: find the eigenpairs $\left(p_{j}^{k}, \lambda_{j}^{k}\right) \in\left\{v_{\mid \Omega_{j}} ; v \in V_{h}\right\} \times \mathbb{R}^{+}$of

$$
a_{\Omega_{j}}\left(p_{j}^{k}, v_{\mid \Omega_{j}}\right)=\lambda_{j}^{k} a_{\Omega_{j}^{\circ}}\left(\Xi_{j}\left(p_{j}^{k}\right), \Xi_{j}\left(v_{\mid \Omega_{j}}\right)\right) \quad \text { for all } v \in V_{h} \text {. }
$$

3. Given a threshold $\mathscr{K}_{j}$ for each $j=1, \ldots, N$, let the GenEO coarse space be defined as

$$
V_{H}:=\operatorname{span}\left\{R_{j}^{\top} \Xi_{j}\left(p_{k}^{j}\right): \lambda_{j}^{k} \leq \mathscr{K}_{j} ; j=1, \ldots, N\right\} .
$$

Assumption: An additional technical assumption is needed for the proof of Theorem 2. In [7] this is given rigorously in the abstract framework but here since we do not go into the details of the proof we will relie on the figure on the right. We assume that given data for the degrees of freedom in the overlap that do not lie on the boundary (i.e. the dots) we can build a discrete harmonic w.r.t. $a_{\Omega_{j}}(\cdot, \cdot)$ extension to the whole of $\Omega_{j}$.

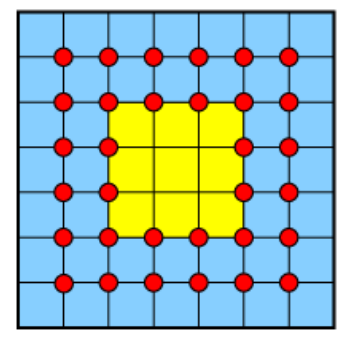

In the next theorem we give our main result which is an estimate for the condition number. It relies solely on the stable splitting property. We provide a suitable 
decomposition that allows to complete the proof along with the main steps of the proof.

Theorem 2 (Stable Splitting and Final Estimate). For any $j=1, \ldots, N$, suppose that the $p_{j}^{k} \in V_{H}$ have been normalized w.r.t. $a_{\Omega_{j}^{\circ}}\left(\Xi_{j}(\cdot), \Xi_{j}(\cdot)\right)$ and let $\Pi_{j}$ be the projection operator: $\Pi_{j}\left(v_{\mid \Omega_{j}}\right)=\sum_{\left\{k: \lambda_{j}^{k} \leq \mathscr{K}_{j}\right\}} a_{\Omega_{j}^{\circ}}\left(\Xi_{j}\left(p_{j}^{k}\right), \Xi_{j}\left(v_{\mid \Omega_{j}}\right)\right) p_{j}^{k}$. Then, for any $v \in V_{h}$, the splitting $z_{H}:=\sum_{j=1}^{N} \Xi_{j}\left(\Pi^{j}\left(v_{\mid \Omega_{j}}\right)\right)$ and $z_{j}:=\Xi_{j}\left(v_{\mid \Omega_{j}}-\Pi^{j}\left(v_{\mid \Omega_{j}}\right)\right)$ satisfies Lemma 1 for $C_{1}=\max _{1 \leq j \leq N}\left(1+\frac{1}{\mathscr{K}_{j}}\right)$ so, by Theorem 1, the condition number of the preconditioned operator is bounded by

$$
\kappa\left(\mathbf{M}_{A S, 2}^{-1} \mathbf{A}\right) \leq\left(1+k_{0}\right)\left[2+k_{0}\left(2 k_{0}+1\right) \max _{1 \leq j \leq N}\left(1+\frac{1}{\mathscr{K}_{j}}\right)\right],
$$

Proof. The only thing that we need to check is $a\left(R_{j}^{\top} z_{j}, R_{j}^{\top} z_{j}\right) \leq\left(1+\frac{1}{\mathscr{K}_{j}}\right) a(v, v)$. Here we only give the key ideas of the proof, the whole proof in a more general setting can be found in [7]. The most important ingredient in the proof is that, because they were obtained through a generalized eigenvalue problem, the $p_{j}^{k}$ form a basis of $\left\{v_{\mid \Omega_{j}} ; v \in V_{h}\right\}$ with the additional orthogonality type properties:

$$
a_{\Omega_{j}^{\circ}}\left(\Xi_{j}\left(p_{j}^{k}\right), \Xi_{j}\left(p_{j}^{l}\right)\right)=0 \quad \text { and } \quad a_{\Omega_{j}}\left(p_{j}^{k}, p_{j}^{l}\right)=0 \quad \text { for all } k \neq l .
$$

Using these properties we obtain

$$
v_{\mid \Omega_{j}}-\Pi^{j}\left(v_{\mid \Omega_{j}}\right)=\sum_{\left\{k: \lambda_{j}^{k}>\mathscr{K}_{j}\right\}} \alpha_{j}^{k} p_{j}^{k} \text {, for any } v_{\mid \Omega_{j}} \text { written as } v_{\mid \Omega_{j}}=\sum_{k} \alpha_{j}^{k} p_{j}^{k},
$$

where the coefficients $\alpha_{j}^{k} \in \mathbb{R}$. Then we make appear the overlap term:

$$
a\left(R_{j}^{\top} z_{j}, R_{j}^{\top} z_{j}\right)=a_{\Omega_{j}}\left(z_{j}, z_{j}\right)=a_{\Omega_{j}^{\circ}}\left(z_{j}, z_{j}\right)+a_{\Omega_{j} \backslash \Omega_{j}^{\circ}}\left(z_{j}, z_{j}\right) .
$$

In the interior $\Omega_{j} \backslash \Omega_{j}^{\circ}$ we have that $\Xi_{j}$ is identity so $z_{j}=v_{\mid \Omega_{j}}-\Pi^{j}\left(v_{\mid \Omega_{j}}\right)$ and because $a_{\Omega_{j} \backslash \Omega_{j}^{\circ}}(\cdot, \cdot) \leq a_{\Omega_{j}}(\cdot, \cdot): a_{\Omega_{j} \backslash \Omega_{j}^{\circ}}\left(z_{j}, z_{j}\right) \leq a_{\Omega_{j}}\left(v_{\mid \Omega_{j}}-\Pi^{j}\left(v_{\mid \Omega_{j}}\right), v_{\mid \Omega_{j}}-\Pi^{j}\left(v_{\mid \Omega_{j}}\right)\right)$. Then by an orthogonality argument $a_{\Omega_{j} \backslash \Omega_{j}^{\circ}}\left(z_{j}, z_{j}\right) \leq a_{\Omega_{j}}\left(v_{\mid \Omega_{j}}, v_{\mid \Omega_{j}}\right)$.

For the other term, we write

$$
\begin{aligned}
a_{\Omega_{j}^{\circ}}\left(z_{j}, z_{j}\right) & =a_{\Omega_{j}^{\circ}}\left(\sum_{\left\{k: \lambda_{j}^{k}>\mathscr{K}_{j}\right\}} \alpha_{j}^{k} \Xi_{j}\left(p_{j}^{k}\right), \sum_{\left\{k: \lambda_{j}^{k}>\mathscr{K}_{j}\right\}} \alpha_{j}^{k} \Xi_{j}\left(p_{j}^{k}\right)\right) \\
& =\sum_{\left\{k: \lambda_{j}^{k}>\mathscr{K}_{j}\right\}} \alpha_{j}^{k^{2}} a_{\Omega_{j}^{\circ}}\left(\Xi_{j}\left(p_{j}^{k}\right), \Xi_{j}\left(p_{j}^{k}\right)\right) \quad \text { (Orthogonality (6)) } \\
& \leq \frac{1}{\mathscr{K}_{j}} \sum_{\left\{k: \lambda_{j}^{k}>\mathscr{K}_{j}\right\}} \alpha_{j}^{k^{2}} a_{\Omega_{j}}\left(p_{j}^{k}, p_{j}^{k}\right) \quad \text { (Definition of eigenproblem (5)) }
\end{aligned}
$$




$$
\leq \frac{1}{\mathscr{K}_{j}} \sum_{\{\text {all } k\}} \alpha_{j}^{k^{2}} a_{\Omega_{j}}\left(p_{j}^{k}, p_{j}^{k}\right)=\frac{1}{\mathscr{K}_{j}} a_{\Omega_{j}}\left(v_{\mid \Omega_{j}}, v_{\mid \Omega_{j}}\right) .
$$

\section{Numerical results}

We run a simulation for the Darcy equation $-\nabla \cdot(\alpha \nabla v)=1$ in $\Omega=[0,1]^{2}$ with homogeneous Dirichlet boundary conditions on the whole of $\partial \Omega$. The mesh is $200 \times 200$ square elements further subdivided into triangles and the finite element discretization uses standard $\mathbb{P}_{1}$ basis functions. All the finite element data is generated using Freefem++ [5]. The coefficient distribution is rather random since it is given by a QR code. This is shown on the left hand side of Figure 1 where in the yellow (or light) parts $\alpha=1$ and in the pink (or dark) parts $\alpha=1000$. The decomposition into subdomains is the 100 subdomain partition obtained via Metis [6] where we add one layer of overlap to each subdomains. This is plotted in the middle of Figure 1. The results are shown on the right hand side of Figure 1 where we have plotted the condition number and the size of the coarse space versus the threshold $\mathscr{K}_{j}$ which is used to select modes for the coarse space. We observe that the coarse space grows roughly linearly with the threshold but the condition number stabilizes quickly. What this illustrates is that there is a good compromise to be found between the size of the coarse space and the efficiency of the method. An automatic optimal choice for $\mathscr{K}_{j}$ is a subject for future research. More thorough numerical experiments can be found in $[7,8]$ including three dimensional examples and results for elasticity.
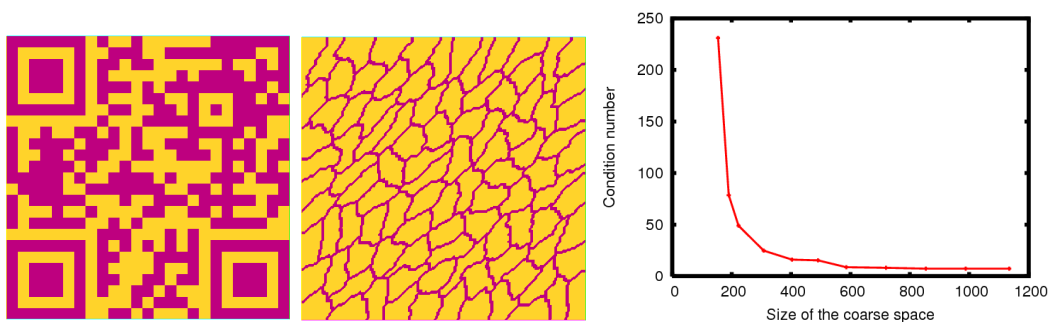

Fig. 1 Left: coefficient distribution (pink or dark is high conductivity) - Middle: Metis partition of the $200 \times 200$ mesh into 100 subdomains - Right: We plot the condition number with respect to the coarse space size when the threshold successively takes the values $\tau \in$ $[0.01 ; 0.05 ; 0.1 ; 0.2 ; 0.3 ; 0.4 ; 0.5 ; 0.6 ; 0.7 ; 0.8 ; 0.9]$. As a matter of comparison: without any coarse space the condition number is 9661 . With just the weighted constant $\Xi_{j}\left(1_{\Omega_{j}}\right)$ per floating subdomain the condition number is 7324: this 64 dimensional coarse space is what we get for GenEO with a barely positive threshold $\tau=0^{+}$(not shown on the graph simply because of scaling issues). We observe that the most troublesome eigenmodes are identified for quite a small value of the threshold and a reasonable size of the coarse space then the condition number stagnates. 


\section{Conclusion}

We have introduced the GenEO coarse space which is a way to automatically make the two level Schwarz method robust. The construction of this coarse space is based on solving generalized eigenvalue problems which isolate good and bad modes in each subdomain. We have presented the steps which lead to the choice of this generalized eigenvalue problem starting with the abstract Schwarz theory and the key ideas of the proof for the condition number estimate. The whole proof and a more general setting can be found in [7]. Although the eigenvalue problems are local, can be solved in parallel and only the smallest eigenvalues are needed, this setup phase could be costly and the study of the overall cost of the algorithm is still work in progress. The related methods in [2, 4] have been extended to a multilevel setting by $[3,12]$. Moreover, this strategy was further applied by some of the authors in the BDD and FETI frameworks [9, 10].

\section{References}

1. Brezina, M., Heberton, C., Mandel, J., Vaněk, P.: An iterative method with convergence rate chosen a priori. Technical report University of Colorado Denver (1999). Earlier version presented at 1998 Copper Mountain Conference on Iterative Methods, April 1998.

2. Efendiev, Y., Galvis, J., Lazarov, R., Willems, J.: Robust domain decomposition preconditioners for abstract symmetric positive definite bilinear forms. ESAIM: Mathematical Modelling and Numerical Analysis 46(05), 1175-1199 (2012)

3. Efendiev, Y., Galvis, J., Vassilevski, P.: Multiscale spectral AMGe solvers for high-contrast flow problems. ISC-Preprint, Texas A\&M University (2012). Submitted.

4. Galvis, J., Efendiev, Y.: Domain decomposition preconditioners for multiscale flows in highcontrast media. Multiscale Model. Simul. 8(4), 1461-1483 (2010)

5. Hecht, F.: FreeFem++, 3rd edn. Numerical Mathematics and Scientific Computation. Laboratoire J.L. Lions, Université Pierre et Marie Curie, http://www.freefem.org/ff++/ (2012)

6. Karypis, G., Kumar, V.: METIS: A Software Package for Partitioning Unstructured Graphs, Partitioning Meshes, and Computing Fill-Reducing Orderings of Sparse Matrices. Department of Computer Science, University of Minnesota, http://glaros.dtc.umn.edu/gkhome/views/metis (1998)

7. Spillane, N., Dolean, V., Hauret, P., Nataf, F., Pechstein, C., Scheichl, R.: Abstract robust coarse spaces for systems of PDEs via generalized eigenproblems in the overlaps. Technical report NuMa, Linz (2011). Submitted.

8. Spillane, N., Dolean, V., Hauret, P., Nataf, F., Pechstein, C., Scheichl, R.: A robust two level domain decomposition preconditioner for systems of PDEs. Comptes Rendus Mathématique 349(23-24), 1255-1259 (2011)

9. Spillane, N., Dolean, V., Hauret, P., Nataf, F., Rixen, D.: Solving generalized eigenvalue problems on the interfaces to build a robust two level FETI method. HAL-00756840 (2012). Submitted.

10. Spillane, N., Rixen, D.: Automatic spectral coarse spaces for robust FETI and BDD algorithms. HAL-00756994 (2012). Submitted.

11. Toselli, A., Widlund, O.B.: Domain decomposition methods-algorithms and theory. Springer-Verlag, Berlin (2005)

12. Willems, J.: Robust multilevel methods for general symmetric positive definite operators. Technical report RICAM, Linz (2012). Submitted. 